\title{
RELAÇÃO DAS OBRAS ENTRADAS NA BIBLIO- TÉCA DA FACULDADE DE DIREITO DE SÃO PAULO, NO PERIODO COMPREENDIDO ENTRE 16 DE FEVEREIRO E 15 DE MAIO DE 1935
}

\section{OBRAS GERAIS (0)}

Almanaque Comercial Brasileiro-1918 -Encad.-1 vol.-Doação.

Anales de la Facultad de Ciencias juridicas y Sociales de la Universidad de La Plata-Tomo VI-La Plata1933-Broch-1 vol.-Permuta.

Annali del Seminario Giuridico della R. Universitá di Palermo-Vol. XVI $\rightarrow$ Cortona-1931-Broch.-1 vol.-Per muta.

Annuario Demografico-vol. I. 1929. S. Paulo, 1932. Encad. 1 vol.-Dloação.

Antenor Nascente-Dicionario etimologico da lingua portuguêsa-Rio de Janeiro-1932. Encad.-1 vol.-Compra.

Arquives de Philasophie du Droit et de Sociologie Juridique $-\mathrm{N}^{\circ}$ s. 3 e 4. -Paris, 1934—Broch.-1 vol.-Compra.

Arquivos de Medicina Legal-Buenos Aires, 1932-Broch.-1 vol.-Doação.

Arquivo da Universidade de Lisboavol. XIII-(Anos de 1928-1931).- Lisboa, 1931-Broch.-1 vol.-Doação.

Anquivo Jadiciario - vol XXXII.-Rio de Janeiro, .1934-Broch-1 vol.Permuta.

Boletim da Bibliotéca Geral da Univergidade de Coimbra-vols. X-XI,Coimbra, 1933-1934.-2 vols.-Permuta
Boletim Mensuel del Seminario de Ciencias Juridicas y Sociales-Buenos Aires, 1934.-Broch.-1 vol.Permuta.

Boletim do Ministerio da Agricultura $\rightarrow$ Rio de Janeiro, 1934 $\_$Broch. -1 vol -Permuta.

Boletim do Ministerio da Educação e Saude Publica-N. ${ }^{\circ}$ s 3-4-Rio de Janeiro, 19:31-Broch.-1 vol.-Permuta.

Boletim da União Panamericana-Washington, 1934-Broch.-1 vol.-Permuta.

Cincoentenario da Estrada de Ferro do Paraná-1885-1935-Encad.-1 vol.Dioação.

Coletaneas de Acordãos-vol. V-Belo Horizonte, 1934-Broch.-1 vol.-Permuta.

Columbia Law Review-New York, 1934 -Broch.-1 vol_-Permuta.

Critica Juridica, Fistorica, Politica $y$ Literaria-Tomo $\mathrm{X}-$ Buenos Aires, 1926-Broch.-1 vol.-Permuta.

Quarta Conferencia Internacional-Buenos Aires, 1910-Enciald.-2 vols.Doação.

Decisões do Superior Tribunal do Estado do Rio Grande do Sul Proferidas Durante o Ano de 1935-Vol. 1-II Porto Alegre, 1933-Brach.-2 vols.Permuta. 
D. N. C.-Revista do Departamento Nacional do Café-Rio de Janeiro, 1934-Broch.-1 vol.-Permuta.

Europe-Revue Mensuelle-Paris, 1934 -Broch.-1 vol-Compra.

Goes Calmon-In Memoriam-Ria de Janeiro, $1933-$ Broch.-1 vol.-Doação.

Homenageando um Apostolo do Direito -Instituto dos Advogados de São Paulo-Doação.

Homenagem a Ricardo Severo-(Discurso)-S. Paulo, 1933-Broch -1 vol. -Doação.

Indicador Alfabetico dos Atos Oficiais Gerais Referentes ao Ministerio da Guerra-Rio de Janetiro, 1934-Broch. 1 vol.-Doação.

Industrias y Finanzas-Revista Tecnica de Bancos y Seguros-Buenos Aires, $1934-$ Broch.-1 vol-Doação.

International Conciliation Documents For The Year 1934-New-York, 1954 -Broch. -1 vol.-Permuta.

Journal du Droit International--Paris, 1934-Broch.-1 vol.-Permuta.

Journal de L'Université des AnnalesParis, 1909-1911-Encad:-4 vols.Doação.

Juarez Tavora- 0 ministerio da Agricultura em 1933-Rio de Janeiro, 1934 -Broch.-1 vol-Compra.

La Giustizia Penale-vol. LX-(Xdella 4a Serie)—Roma, 1934-Broch.-1 vol. Permuta.

L'Annuaire de Paris "Comercial"-Paris, 1933-Encad:-1 vol-Doação.

Larousse du XXe. Siécle-Paris-Encad. $\longrightarrow 6$ vols. -Compra.

Le Mois-Synthése de l'activité mondiale-Paris, Mars $\dashv$ Avril, 1935-ABroch ${ }^{*}$

-1 vol.-Compra.

L'Esprit International-Paris, 1934Broch.-1 vol -Permuta.

Memoria Historica da Faculdade de Direito do Recife-Recife, 1856-1883.Encad-2 vol-Doação

Nelson de Rezende - 0 Departamento de Estradas de Rodagem-A minha
Atuação-S. Paulo, 1933-Broch.-1 vol-Doação.

Noel-Revista mensal-Rio de Janeiro, 1926—Broch.—1 vol —Doação.

Paraná Judiciario-Vol. XX-Curitiba, 1934-Broch -1 vol.-Permuta.

Plinio Ayrosa-Dicionario portunguêsbrasiliano e brasiliano-português.S. Paulo, $1934-$ Broch.-1 vol.-Doação.

Political Science Quarterly-Vol. XLIX $\rightarrow$ Niew-York, 1934—Broch.-1 vol.Compra.

Revista de Jurisprudencia Brasileiracial da Sociedade Brasileira de Criminologia-Vol. IV-Rio de Janeiro, 19,34-Broch.-1 vol-Permuta.

Revista de Jurisprudencia BrasileiraRio de Waneiro, 1934-1935-Broch.3 vols.-Permuta.

Revista del Colegio de Abogados de Buenos Aires-Buenos Aires, 1934Broch.-1 vol.-Permuta.

Revista do Foro-Vol. XXIX-Paraiba, 1933-Broch:-1 vol.-Dermuta.

Revista do Instituto Historico e Geografico do Espirito Santo-Vitoria, 1934-Broch.-1 vol-Permuta.

Revista do Superior Tribunal Federal -Vols. 48 a 8i3 $\longrightarrow$ Rio de Janeiro,-19231925-Broch,-37 vols.-Compra.

Revista dos Tribunaes-V.ol. XXVBaia, 1933-Broch-1 vol.-Permuta.

Revista Militar Brasileira-Vol. XXXII Rio de Janeira, 1933-Broch.-1 vol. -Doação.

Revue Critique de Droit International Tome XXIX-Paris, 1934-Broch.-1 vol.-Compra.

Revue de Droit Penal et de Criminologie-Bruxclles, 1934-Broch.-1 vol.Compra.

Sales Filho-Programa Nacional-(Radio Difusão) — Rio de Janeiro, 1934Broch-1 vol.-Droação،

Sales Filho-Tres anos de administração—Rio de Janeiro, 1935—Broch.1 vol.-Doação.

The Annals-Filadelfia, January, 1935 $\rightarrow$ Broch.-1 vol -CCompra. 
The Annals of The American Academy of Political and Social Science-Filadelfia March, 1935-Broch.-1 vol.Compra.

\section{FILOSOFIA (1)}

Compyré (Gabriell)-Psychologie appliquée a l'éducation-Paris-Encad-1 vol.-Doação.

Fouillet $(\mathrm{H} ;)$-A Vida Sexual na Russia Sovietica-Rio, 1934-Broch.-1 vol.-Dqação.

Herzen, Alexandre-Le Cerveau et l'activité cérébrale-Paris, 1887-Encad -1 vol.-Doação.

Letourneau, Ch_L'Evolution de la morale-Paris, 1887-Encad.-1 vol.Doação.

Littré-Auguste Comte et la Philosophie Positive-Paris, 1864-Encad1 vol.-Doação.

Marestan, Jean-L'Education Sexuelle -Paris, 1913-Encad.-1 vol--Doação.

M. Carlos-Aspectos da Filosofia Universal-Rio de Janeiro, '1935-Broch. 1 vol.-Doação.

o Problema Sexual-Rio de Janeiro, 1913-Broch-1 vol_-Doação.

Paulhan, Fr.-Les phénoménes affectifs-Paris, 1887-Encad.-1 vol.Doação.

Rig, Julds-La Philosophie PositiveParis, 1880-Encad.-2 vols-Doação

Roberty, E. de-Auguste Conte et Herbert Spencer-Paris, 1894-Encad.1 vol -Doação.

Van Acker, L.-Introdução á Filosofia Logica-S. Paulo, 1932-Encad.-1 vol. -Compra.

Véran, Eugéne-La morale-Paris, 1884 -Encad-1 vol_-Doação.

\section{RELIGLÃO (2)}

Carlos Peixoto de Alencar-Roteiro dos Bispados do Brasil-Ceará, 1864-Encad-1 vol.-Permutado.
José Carlos de Macedo Soares-Le Cardinalat-(Conferencia)_Paris, 1932 $\longrightarrow$ Broch -1 vol.-Doação.

José do Carmo Barata-Historia Eclesiastica de Pernambuco-Recife, 1922 -Broch.-1 vol.-Permutado.

Letourneau, Ch.-L'Évolution religieuse dans les diverses races humaines -Panis, 1892-Encad.-1 vol.-Doação

Lino de Monte Carmelo Luna-Memoria historica e biografia do Clero de Pernambuco-Pernambuco, 1857-Encad. 1 vol.-Penmutado.

\section{SOC. DIREITO (3)}

A Academia Brasileira de Letras e Amelia de Freitas Bevilaqua-Rio de Janeiro, 1930—Broch.-1 vol.-Doação.

Albert, Charles-L'Etat moderne, ses principes et ses institutions-Paris, 1929—Broch-1 vol.-Doação.

A. de Sampaio Doria-Habeas-Corpus —São Paulo, 1925-Broch.-1 vol.Doação.

A. F. de Melo Morais-O Brasil Social e Politico-Rio de Janeiro. 1872Encad.-1 vol.--Doação.

Alfredo Pujol e Clovis Ribeiro-Do Protesto das Duplicatas-São Paulo, 1924-Broch -1 vol-Doação.

Almachio Diniz-Das ações de despejo em face do moderno direito civilRio de aJneiro, 1922-Encad.-1 vol. -Doação.

Atavilla, Eurico-Teoria soggetiva del reato-Napoli, 1933-Broch.-1 vol.Doação.

Atvilla, Eurico-Lineamenti di diritto criminale-Napoli, $\quad$ 1932-Broch.-1 vol.-Doação.

Atavilla, Eurico-Ís Suicidio nella psicologia, nclla indagine giudiziaria e nel diritto.-Napoli, 1932-Broch.-1 vol.-Doação.

Amaro Cavalcanti-Politica e finanças -Rio de Janeiro, 1892 Encad.-1 vol. -Doação.

Antokoletz, Daniel-Tratado de Derecho Constitucional y Administrativo- 
Buenos Aires, 1933-Broch.-2 vols.Compra.

A. Pompeo-A defesa do Brasil-(profissão de fé integralista)-S. Paulo, 1935-Broch-1 vol.-Doação.

Araujo Castro--Manual da Constituição Brasileira-Rio de Janeliro, 1918Broch.-1 vol.-Doação.

Arduino, Félix $\mathbf{E}-$ La pasividad-estudio comparado sobre seguros sociales, jubilaciones y pensiones-Buenos Aires, 1927-Broch.-1 vol.-Permuta.

Armando Costa-Livramento condicional-IRio, 1934-Broch -1 vol._-Com pra.

Asua, Luis Jimenez de-Casos de Derecho Penal-Madrid, 1929-Broch.1 vol.-Compra.

Atos Internacionais Vigentes no Brasil - Ministerio das Relações Exteriores -Rio de Janeiro, 1927-Broch.-1 vol -Dloação.

Axelrod, Alexandre-L'Oeuvre economiqud des Soviets-Paris-Broch.-1 vol.-Doaçãalo.

Barbie, Léon-Guide Pratique des divorce-Paris, 1927-Encad-1 volDoação

Benoiste, Charles-Les malladies de la démocratie-Paris, 1929-Broch.-1 vol.-Doação.

Bontandini, V.irginio-La Cambiale e altri effetti de comercio-MilanoEncad.-1 vol.-Doação.

Breno Viana-Cartatechnologia mercantil-Rio de Uaneiro, 1927-Encad. -1 vol.-Doação.

Calamandrei, Piero-Studi sul processo civile-Padova, $1930-$ Broch-1 vol.Compra.

Cambon, Vitor-L'Alemagne nouvelle -Paris-Encad-1 vol.-Doação.

Candidatos do Partido Constitucionalista-São Paullo, 1934-Broch-1 vol. Doação.

Candido de Oliveira Filho-Lei do inquilinato-Rio de Janeiro, 1922Encad,-1 val.-Doação.

Carone, s. J-Raciocinio- 0 remedio
social-S. Paulo, 1935—Broch.-1 vol. -Doarão.

Castro, Horacio de-Principios de Derenho Sovietico-Madrid, 1931-Broch -1 vol-Permuta.

Castro, Mario de-Para uma nova civilisaçãa-Lisboa, 1933-Broch-1 vol. -Doação.

Chéron, Lucien-Les Causes de divorce et de séparation ide corps-Paris, 1934-Broch.-1 vol.-Compra.

Chonzal, Conego Bernarda-Reficidio e Regnicidio-O Crime do Ferreira do Paço um ano depois-Lisboa, 1909 -Encad.-1 vol.-Permutado.

Clovis Bevilaqua-Codiga Civil dos Estados Unidos do Brasil, comentado -Vol. V-Rio de aJneiro, 19:34Broch.-1 vol.-Doação.

Codigo Penal de La Nacion Argentina $\rightarrow$ Buenos Aires, 1922-Broch.-1 vol. -Permuta.

Coleçãa de Atos Internacionais-Ministeruio das Rellações Exteriores_Fases de $n^{\circ} 30$ a $60-$ Rio de Janeiro, 19301934-Broch.—1 vol.-Doação.

Colecion Completa de Las Sentencias Dictadas por el Tribunal SupremoTomo VI—Ano 1933-Madrid, 1934Broch. -1 vol.-Permuta.

Contuzzi, F. P.-Diritto Internazionale Privato-Milano, 1911-Encad-1 vol. 一Doação.

Coquelin, Charles-Le credit et des Banques-Paris, 1876-Encad.-1 vol. -Doação.

Coraça, Bento de Jesus-A cultura integral do individuo-Lisboa, 1933Broch.-1 vol.-Doação.

Coste, Adolphe-Nouvel exposé d'économie politique et de physiologier sociale-Paris, 1889-Encad.-1 vol.Doação.

Coste, Adolphe-Les questions sociales contemporaines-Paris, 1886-Encad.-1 vol.-Doação.

Coste, Adolpre-Hygiéne sociale contre le paupérismci-Paris, 1882-Enc. -1 vol.-Doação.

Courcelle-Seneuil J. G.-Traité Théorique et pratique des opérations de 
Banque-Paris, 1876-Encad.-1 vol. $\longrightarrow$ Doação.

Courtis, Fils Alph.-Traité élementaine des operations de Bourse ct de Change-Paris-Encad.-1 vol.-Doação.

Courtois, Allph.-Histoire des Banques en France-Paris, 1881-Encad.-1 vol.-Doação.

Covarruvias-A' Leyna Totelani Episcopi Segoviensis Juisconsulti Celeberrimi...-Lugduni, 1568-Encad.1 vol.-Doacão.

Crépon, F.-De la 'négociation des effets publies \& autres.-Paris, 1886Encad-1 vol.-Doação.

Crowell-Republies-Encad.-1 vol.Doą̧ão.

Cursino Belem-Finalidade do direito a do anarquismo social-Ceará-Fortaleza, 1934-Broch-1 vol.-Dolação.

Cuzzi, Mario-Le sevitu dalle distanze legali secondo il nostro codice civile -Torino, 1923—Broch. -1 vol.- Doação.

Daubresse, Louis-Assurances maritimes-Belgique - Broch. -1 vol.-Doação.

Daubresse, Louis-Les Ventes Commercialds-Belgique-Broch-1 vol. Doação.

Decreta de 5 de Diciembre de 1933 Aprobando el Reglamento de Verificaciones electricas $y$ de Regularidad en el Suministro de EnergiaMadrid, 1934-Broch.-1 vol.-Permuta.

Del Vecchio, Giorgio-Etica, Diritto e Stato-Roma, 1934-Broch.-1 vol.Doação.

Desqueyrat, A.-L'Institution, le droit objective et la tecnique positiveBordeaux, 1933 -Broch.-1 vol_- Permuta.

Diego, F. Clementci de-Instituciones de Derecho Civil Espanol.-Madrid, 1930-Broch-13 vols.-Compra.

Dilermando Cruz-Reforma da lei de Falencia-(Estudo modificativo da lei 2.024)—Rio, 1928-Broch.-1 vol.Doação.
Donnat, M. eun-L'Etat de Dalifornie -Parisi, 1878-Encad.-1 vol.-Doação.

Donnat, M. Leon-L'election $d \epsilon$ is fonctionnaires publics-Paris, 1878Encad.-1 vol.-Doação.

Durand, Louis-Le Crédit agricole en en France et l'Etranger-Paris, 1891 -Encad.-1 vol.-Doaçãio.

Elysio de Carvalho-Brasil, potencia mundial-Rio de Janeiro, 1919-Broc. -1 vol.-Doação.

Eusebio de Queiroz Lima-Teoria de Estada-Rio de Janeiro, 1930-Encadernado-1 vol.-Compra.

Francisco Eugenio do Amaral-A moratoria e o decreto de emissão de papel moeda-S. Paulo,-1914. Broch. -1 vol.-Doação.

F. F. de Sousa Reis-A depressão comercial e o Funding Loan de 1931Rio de Ulaneiro, 1924—Broch.-1 vol. -Doação.

Fuss, Henri-La prévention du chomage et la stabilisation économiqueBruxelles, 1925-Broch.-1 vol-Doação.

Gabriel L. Bernardes e R. Machado Bittencourt-Apelação civil $n .^{\circ} 3.183$ Rio de Janeiro, 1933-Broch-1 vol. -Doação.

Gachon, Jean-La Politique Etrantére des Etats-Unis-Paris, 1929-Broch.1 vol.-Doação.

Garófalo, Pietro-Principios de Derecho Constitucional-Madrid, 1934Broch-1 vol.-Compra.

Gaudement, Jean-Etude sur le régime juridique de l'indivision en Droit Romain-Paris, 1924-Broch-I vol. -Compra.

Gentijon, Paul-Rome sous le faiscear -Paris-Broch-1 vol.-Doação.

Gini, Corrado-Curso de estatisticaBarcelona, 1935-Encad-1 volCompra.

Godart, M. Emile-Manuel juridique des vices rédhibitoires-Paris, 1923 -Encad.-1 vol.-Doação.

Haller, Marquerite-Essai sur l'influence du fait et de la faute de la vieti- 
me sur sou droit a réparation-Paris, 1926-Broch.-1 vol.-Doação.

Helio Lobo-A's portas da guerra-Rio de Janeiro, 1916-Encad.-1 vol.Doação.

Helio Lobo-Cousas diplomaticas-Rio, 1918-Encad.-1 vol.-Doação.

Horacio Gonçalves Pereira-Recursos Municipais-S. Paulo, 1925-Broch.2 vols.-Doação.

Horn, J. C.-La liberté des BanquesParis, 1926—Encad.-1 vol.-Doação.

Idelfonso Simões Lopes Filho-Defendendo meu pae-Porto Alegre, 1935 -Broch.-1 vol.-Doação.

Issaurat, C.-La pédagogie son évolution et son histoire-Paris, 1886Encad.-1 vol.-Doação.

João da Gama Cerqueira-A questão "Mousseline"-S. Paulo, 1935-Broch. -1 vol.-Doação.

João da Gama Cerqueira-Recurso Crime-Razões de recurso-S. Paulo, 1935-Broch.-1 vol.—D'oação.

J. de Oliveira-Pareceres-S. Paulo, 1934-Broch.—1 vol.-Doação.

Jaquotot, José Ma. del Sol-La accion de privilegio de cuenta jurada de Abogados y Procuradores contra los Sindicos de quiebros y ConcursosMadrid, 1933-Broch.-1 vol.-Doação.

José Carlos da Silva Freire-Principios de economia politica- Caçapava, 1917—Encad.-1 vol.—Doação.

Kerdaniel, Edouard L. de-Les Animaux en Justice-Paris, 1925-Broch.-1 vol.-Doação.

Kuntz, Roberto-La verdad sobre Estados Unidos-Buenos iAires-Broch. -1 vol.-Permuta.

Laveleye, Emile de-La monnaie et le bimétallisme internacional-Paris, 1891-Encad.-1 vol.-Doação.

Lavisse, Ernest-L'Histoire pollitique de L'Europe-Paris, 1890-Encad.-1 vol.-Doação.

Lo Bon, Gustave-Les lois psychologiques de L'Evolution des peuplesParis, 1894-Encad.-1 vol.-Doação.
Le Probléme des Souces du Droit Positif-Paris, 1934-Broch.-1 vol.Compra.

Leray, René-L'arbitrage et la loi nouvelle sur la clause compromissoire-Paris, 1927-Broch.-1 vol.Do|ação.

Letourneau, Ch.-L'evolution du mariage et de la famille.-Paris, 1888Encard.-1 vol.-Doação.

Letourneau, Ch.-L'Evolution politique dans les diverses races humainesPartis, 1890-Encad.-1 vol.-Doação.

Letourneau, Ch.-L'evolution de l'éducation dans les diverses races humaines, Paris, 1898-Encad.-1 vol.Dłolação.

Letourneau, Ch.-La guerre dans les diverses races humaines-Paris, $\mathbf{1 8 9 5}$ - Encad.—1 vol.-Doação.

Letourneau, Ch.-L'évolution de l'esclavage dans les diverses races humaines-Paris, 1897--Encad.-1 vol. -Doação.

Letourneau, Ch.-L'évolution de la proprieté-Paris, 1889-Encad.-1 vol.Doação.

Letourneau, Charles- La sociologieParis, 1880-Encad.-1 vol.-Dopação.

Letourneau, Charles-L'évolution juridique dans les diverses races humaines-Paris, 1891-Broch.-1 vol.Doação.

Macgregor, John-The Constitution of England - London, 1853 - Encad.-1 vol.-Doação.

Madray, Gilbert-De la répresentation en droit privé-Paris, 1931-Broch.1 vol.-Compra.

Mallet, Paule-La renonciation a succession-Paris, 1931-Broch.-1 vol. -Compra.

Manoel da Silva Carneiro-Reivindicação disfarçada em demarcação-S. Paulo, 1930-Broch.-1 vol.-Doação.

Maria Palancar y Eugenio Peres Botifa - La prevencion de los accidents del trabajo-Madrid, 1934-Broch.-1 vol. -Compra. 
Massari, Eduardo-La norma penaleNapoli, 1913-Broch.-1 vol.-Permutado.

Molinari, G. de-Les Bouses du Travail-Paris, $1893-$ Encad.-1 vol.

Monteiro, Domingos-A crise de idealismo na arte e na vida social-Lisboa, 1933-Broch.-1 vol.-Doação.

Nelson de Rezende-Prefieito tecnico ou Prefeito politico? (Separata da Revista Pol.)-S. Paulo, 1933-Broch.1 vol.—Doação.

N. Tolentino Gonzaga-Interditos possessorios —Rio dei Janeiro, 1923 Encad.-1 vol.-Doação.

Otavio Tarquinio de Sousa-A mentalidade da Constituinte ( 3 de Maio a 12 de Novembro de 1923)-Rio de Janeiro, 1931-Broch-1 vol.-Doação.

Otto Gil-As leis da Russia Soviets perante a Justiça Brasileira-Rio de (Jianeiro, 1932-Broch.-1 vol.-Doação.

Pedro de Melo-A questão social brasileira e a sua solução racional $\longrightarrow$ S. Paulo, 19;33--|Broch.-1 vol.-Doação.

Peret, P.-La Question ouvriére- Paris, 1893-Encad.-1 vol.-Doação.

Pernambuco Filho e Adauto BotelhoVicios sociais elegantes- Rio de Janeiro, 1924-Encad.-1 vol.-Doação.

Pierre, André-La Féderation Soviétique et ses Républiques-Paris, 1932 -Broch.-1 vol.-Doação.

Pierre, Eugéne-Trait de Droit Politique Electoral et Parlamentaire- $\mathrm{Pa}$ ris, 1893-Encad.-1 vol.-Doação.

Politis, Nicolas-Les nouvelles tendances du droit international-ParisBroch.-1 vol.-Permuta.

Politis, Nicolas-La neutralité et la Paix - Paris-Broch.-1 vol.-Permuta.

Pontes de Miranda-Tratado de Direito Internacional Privado-Rio de Janeiro, 1935-Broch.-2 vols.-Compra.

Programmes. Conservatoire National des Arts et Métiers.-Paris, 1930.Broch.-1 vol.-Dloação.
Programas do Curso de Bacharelandos para 1935-S. Paulo, 1935-Broch.1 vol.-Doação.

Projecto do Codigo Civil BrasileiroRio de Janeiro, 1902—Encad.-8 vols. -Doação.

Projecto de Reforma a los Planes de Estudio de la Enseñansa MediaBuenos Aires, 1934-Broch.-1 vol.Permuta.

Raul Humberto de Lima Simões-Credito bancario-Lisboa, 1930-Encad. -1 vol.-Doação.

Renato Maia-Cartilha do comerciante $\longrightarrow$ S. Paulo, 1925-Broch.-1 vol.Dọação.

Riper, Georges-La régle morale dans les obligations civiles-Paris, 1935Broch.-1 vol.-Compra.

Rittershansen, Henrich-Paro forzoso y capital-Baroelona, 1935-Encad.1 vol. $\longrightarrow$ Compra.

Say, M. Léon-Les solutions démocratiques-Paris, 1886-Encad.-2 vols. -Doação.

Salgueiro, Eduardo- 0 suicidio dos catedraticos del Coimbra-Lisboa, 1933 - Broch.-1 vol.-Doação.

Schmidt, Carl-Téorie de la Constitucion-Madrid, 1927-Broch.-1 vol.Compra.

Sebaut, A. - L'impot - Tunis, 1891 Encad.-1 vol.-Doação.

Séché, Alphonse-Le Dictatieur ou l'homme de la République-Paris, 1933-Broch.-1 vol.-Doação.

Severino Prestes-Lições de Direito Criminal-Rio de Janeiro, 1897Encad.-1 vol.-Doação.

Sisto, A.-Diritto Marittimo ItalianoMilano, 1906-IEncad.-1 vol.-Doação.

Sousa Diniz-Docimasie Sialique-S. Paulo, 1927-1 vol.-Dopação.

Spear, Samuel F.-Religion and the State-New York, 1876 - Encad. - 1 vol.-Doação.

Spencer, Herbert-Principes de sociologie-Paris, 1882-Encad.-2 vols.Doação. 
Stearns, Charles W.-Constitution of the United States of America-New York, 1873-Broch.-1 vol.-Doação.

Tedeschi, Felice-Del metodo nello studio del diritto civil€-Torino, 1877Encad.-1 val.-Doação.

Terry, J. Morell y-Comentarios a la Legisiação Hipotecaria-Tomo VMadrid, 1934-Broch.-1 vol.-Permuta.

Tuhr, A. Von-Tratato de las obligaciones-Tomo II-Madrid, 1934Broch.-1 voll--Permuta.

Vabres, H. Donnedieu de-La justice penale d'laujourd'hui-Paris, 1929Encad.-1 vol.-Doação.

Van Ryn, Jean-Responsabilité aquilienne et contrats en dropit positifParis, 1932-Broch.-1 vol.-Compra.

Verges, Pierre-La conversion de la séparation de corps en divorce-Paris, $1934 \longrightarrow$ Broch.-1 vol.-Compra.

Vernęau, R.-L'Hormme, racès et coutumes-Larousse-Paris-is'/d. -Encad. 1 vol.-Compra.

Vicano, José M. G. de Echavarri yComentarios al Codigo de Comercio -Valladolid, 1933-Broch. -5 vols.Permuta.

Vicente Azevedo-Ação de indenisação -Dlanos praticados durante a revoluçã'o de 1930-Responsabilidade Civil do Estada-Defesa do rerario publico-S. Paulo, 1935-Broch.-1 vol. -Doação.

Viforeaun, C. Pierre-Contribuition a l'étude du contrat dans le projet Franco-Italien et en droit comparéParis, 1932-Broch. $\rightarrow 1$ vol.-Permuta.

Vilela, A. de-A Universidadé falou !... Figueira da Fóz, 1933-Broch.-1 vol. -Doação.

Vilela, A. Lobo-A crise da Universidade-Figueira da Fóz, 1933—Broch. -1 vol.-Doação.

Wambangh, Sarah- Plebiscites Singe the World War.-Washington, 1933 - Encad.-2 vols.-Permuta.

White, M. Dupont-La centralisation suit a l'individu at l'État-Paris, 1861 -Encad.-1 vol.—Doação.

\section{FJLOLOGLA - (4)}

Hovelacque, Abel-La linguistique-Paris, 1881.-Encad.-1 vol.-Doração.

Holmes, F.-Grammar of the english language.-New York, 1871-Encad. 1 vol.-Doação.

Mario Barreto-Através do Dicionario - da Gramatica.-Rio de Janeiro, 1927-Broch.—1 vol.-Doação.

\section{CIencIas PURAS - (5)}

Barker, George F.-Elementary Chemistry-New York, 1872—Encad.-1 vol.-Doação.

Beaunis, $\mathbf{H}-\longrightarrow \mathrm{Phy}$ hiologie humaine- $\mathrm{Pa}-$ ris, 1888-Encad-1 vol.-Doação.

Berget, Alphonse - L'Air - Larousse Paris, s/d.-Encad.-1 vol.-Compra.

Cazin, A.-L'etincelle életrique-Paris, 1880-Encad.-1 vol.-Doação.

Clowes, Frank-Practical ChemistryPhiladelphia, 1877-Broch. -1 vol.Doação.

Humboldt, Alexandre-Cosmos-Essai d'une description physique du Monde-Bruxelles, 1852-Encad.-3 vols. $\longrightarrow$ Doação.

La science, ses progrés, ses applications - Larousse - Paris, s/d.-Encad.-2 vols.-Compra.

Letourneau, Charles-La Biologie-Paris, 1877-Encad.-1 vol.-Doação.

Pileur, A. Le-Le Corps humainesParis, 1877-Encad.-1 vol.-Doação.

Robin, Aug.-La Terre-Larousse-Paris, s/d.-Encad.-1 vol.-Compra.

Topenard, Paul-L'Anthropologie-Paris, 1879-Encad-1 vol.-Dopăåo.

Watts, W. Marshall-Organic Chemistry-New York, 1873-Encad.-1 vol. -Doação.

\section{CUENCIAS APLICADAS - (6)}

A. Austregesilo- A cura dos nervosos (conselhos madicos) - Rio de Janeiro, 1918-Encad.-1 vol.-Doação. 
Bordier, A.-La Géographie médicaleParis, 1884 -Encad.-1 vol.-Doação.

Florestas e Silvicultura nos Estados Unidos $\longrightarrow$ Ministerio da AgriculturaWashington, 1922-Broch.-1 vol.Droação.

Héring, C.-Médicine homoeopathique domestique-Paris, 1891-Encad.-1 vol.-Diqação.

Jaquerod- 0 tratamento higienico e dictetico da tuberculose pulmonarLyon, 1928-Encad.-1 vol.-Doação.

Larbalétrier, Albert-L'Agriculture et la soience agronomique-Paris, 1888 -Encard.-1 vol.-Doação.

Maia, Samuel-Elogio do vinho- Lisboa, 19332-Broch.-1 vol.-Doação.

Marden, Orison Swett-La iniation en los negocios-Barcelona, 1919—Encad. -1 vol.-Doação.

\section{BELAS ARTES - (7)}

L'Art des origines a nos jours-Larousse-Paris, s/d.-Encad.-2 vols.Compra.

\section{LITERATURA -}

Aloisio de Castro-Rimario-1926Broch.-1 vol.-Doação.

Antonio Correa d'Oliveira-Auto das quartro estações-Porto, 1928.-Broch. 1 vol.-Doação.

Armando de Arruda Pereira-Heróes abandonados! —S. Paulo, 1925-Broch. -1 vol. -Doação.

Boileau-Satires- Paris, 1885-Encad. -1. vol.-Doação.

Clovis Amorim-O Alambique (Romance)-Rio de Janeiro, 1934-Broch.1 vol.-Doação.

Conde de Sabugosa-Bobos da CorteLisboa, 1924-Broch.-1 vol.-Doação.

D'Annunzio Giabriele-La Nave-Milano, 1908-Broxch.-1 vol.-Doação.

Diderot-Romans et contes-Paros, 1887 -Enicad.-3 vols.-Doação.
Didi Caillet-Taú (Contos)-Rio de Jandiro, 1932-Broch.-1 vol.-Doação.

Fontes, Amando- Os Corumbas-Rio de Jantiro, 1933-Broch.-1 vol.-Doação.

Francisci, Pietro de-Ai Giuristi italiani-Discorso-Roma, 1932-Broch. -1 vol.--Doação.

Garção, P. A. Correa-Obras poeticas e oratoria-Roma, 1888-Encad.-2 vols. -Doação.

Grave, João-Os famintos (Episodios da vida popular) $\longrightarrow$ Porto, 1903-Encad.1 vol.

Histoire de la literature françaiseLarousse-Paris, s/d._Encad._-2 vols. -Compra.

Jorge Amado-Cacáu-Rio de Janeiro, 1934 - Broch.-1 vol.-Doação.

José Euclides-Ensaios e conferencias $\longrightarrow$ Paraiba, 1922-Broch.-1 vol.-Doação.

Le Barbier de Séville-Théatre de Beaumauchais-Paris, 1888-Encad.1 vol.-Doaçäo.

Loopardi, Giacomo-Poemas-S. Paulo, 1934-Encad.-1 vol.-Doação.

Letourneau, Charles-L'évolution littéraire dans les diverses races humaines-Paris, 1894 -Encad.-1 vol.Doação.

Levi Carneiro-Discurso-Rio de Janeiro-Broch-1 vol.-Doação.

Lopes, Oscar - Três conferemcias - A tentação-O dia e a noite-Porto, 1912-Encad.-1 vol.-Doração.

Lourenço Filho-Joazeiro do Padre Cicero-S. Paulo-Broch.-1 vol.-Doação.

Medeiros e Albuquerque $\rightarrow$ Fim-S. Paulo-Broch.-1 vol.-Doação.

Millanvoye, Bertrande-Anthologie des poétes de Montmartre-Paris-Encad. -1 vol.-Permutado.

René Sand e outros-L'examen médical en vue du mariage-Paris, 1927Broch.-1 vol.-Doação.

Rodrigues de Carvalho-Cancioneiro do Norte—Paraiba do Norte, 1928Broch.-1 vol.-Doação. 
Romero, Silvio-Martins Pena-Porto, 1901-Encad.-1 vol.-Permutado.

Saint Pierre, Bernardine-Paul et Virginie-Paris, 1888-Encad.-1 vol.Doação.

Sergie Milliet-Roberto-Narrativa-S. Paulo, 1935-Broch.-1 vol.-Doação.

Thátre de Moliére-Paris, 1889-Encad. 1 vol.-Doação.

Vargas Neto-Joá-Porto Alelgre, 1927 $\rightarrow$ Broch.-1 vol.-Doação.

Vicente de Carvalho-Poemas e Canções-1908-Encad.-1 vol.-Doação.

Viegas, Artur-O poeta Santa Rita Durão-bruxelles-Paris, 1914—Broch.1 voll.-Permutado.

\section{HISTORIA E GEOGRAFIA - (9)}

Almeida, Antonio José de-Quarenta anos de vida literaria e politicaLisboa, 1933-Broch.-1 vol.-Doação.

Asua, Luis Jiménez de-Un viage al Brasil—Madrid, 1929—Broch.-1 vol. $\rightarrow$ Doação.

Coubertin, Pierre de-L'évolution Francaise sous la troisiéme RépubliqueParis, 1896-Encad.-1 vol.-Doação.

Curatulo, G. Emilio-Anita GaribaldiMilano-Romal, 1932-Broch.-1 vol. -Doação.

Felipe Francisco Pereira-Roteiro da costa do norte do Brasil-1875Encad.-1 vol.-Permutado.

Felix Pacheco-Robles e CogumelosJosé do Patrocinio e os pigmeus da
imprensa-Rio de Janeiro. 1932Broch.-1 vol.-Doação.

Henrique Celho-Joaquim Nabuco e Castno Alves-S. Paulo, 1923,-Broch. -1 vol.- Daação.

Joaquim Daniel Lacerda-Curso de Historia Universal-Rio de Janeiro, 1885 - Encad.-1 vol. $\rightarrow$ Dioação.

José Torres de Oliveira-Anchieta e a Pacificação dos indios_LS. Paulo, 1933 -Broch.-1 vol.-Doação.

Jousset, P.-L'Espagne et le Portugal illustré-Larousse-Paris, s/d.Encad.-1 vol.-Compra.

Lawrence, Eugene-Historical Studies -New York, 1876-Encad.-1 vol.Doação.

Lopes Gonçalves-O Amazonas-New York, 1904-Encad.-1 vol.-Doação.

Ludwig,Emil -Le monde tel que jé l'ai vu-Paris, 1932-Broch.-1 vol.-Doação.

Maréchal, E.-Histoire contemporaines -Paris-Encad.-1 vol.-Doação.

Mario Melo-Frei Caneca-Recife, 1933 - Broch. 1 vol.-Doação.

Pedro Parley-Historia Universal-Rio de Janeiro, 1884-Encad.-1 vol.Doação.

Resquin, Francisco Isidoro-Datos historicos de la guerra del ParaguayBuenos LAires, 1895-Encad.-1 vol.Permutadio.

Sarfatti, Margherita G.-Dux-Verona, 1932-Broch.-1 vol.-Doação.

Vogel, Charles-L'Europe OrientaleParis, 1881-Encad.-1 vol.-Doação. 